\title{
Hemi-Section: A Ray of Hope for Badly Broken Tooth
}

\author{
Dr. Kamal Nabhi ${ }^{1}$, Dr. Ajay Chhabra ${ }^{2}$, Dr. Varun Jindal ${ }^{3}$, Dr. Simran Pal Singh ${ }^{4}$ \\ ${ }^{1}$ Post Graduate student, Department of Conservative Dentistry and Endodontics, Bhojia Dental College and Hospital, Bhud, Baddi, Distt \\ Solan, Himachal Pradesh. \\ ${ }^{2}$ Principal, Professor and Head, Department of Conservative Dentistry and Endodontics, Bhojia Dental College and Hospital, Bhud, Baddi,
} Distt Solan, Himachal Pradesh. ${ }^{3}$ Reader, Department of Conservative Dentistry and Endodontics, Bhojia Dental College and Hospital, Bhud, Baddi, Distt Solan, Himachal
Pradesh.

${ }^{4}$ Post Graduate student, Department of Conservative Dentistry and Endodontics, Bhojia Dental College and Hospital, Bhud, Baddi, Distt Solan, Himachal Pradesh

\begin{abstract}
The management, treatment and long-term retention of mandibular molar teeth with furcation involvement have always been a challenge in periodontal therapy. Hemisection has been used successfully to retain teeth with furcation involvement. Hemisection represents a form of conservative procedure, which aims at retaining as much of the original tooth structure as possible. It may be a suitable alternative to extraction. Hemisection of the affected tooth allows the preservation of tooth structure, alveolar bone and cost savings (time and money) over other treatment options. The preservation of posterior abutment teeth permits oral rehabilitation with fixed bridges instead of removable prosthesis. The keys to long term success appear to be thorough diagnosis followed by interdisciplinary approach with endodontic, surgical and prosthetic procedures. Preservation of a hopeless tooth is possible by selecting patients with good oral hygiene, and careful surgical and restorative management. This case report describes a simple procedure of hemisection in a mandibular molar with socket preservation and subsequent restoration of the tooth with fixed prothesis.
\end{abstract}

Keywords: Mandibular Molars, Furcation Involvement, Hemisection, Restorative, Endodontic

\section{Introduction}

Modern advances in all phases of dentistry have provided the opportunity for patients to maintain a functional dentition for lifetime. Therapeutic measures performed to ensure retention of teeth vary in complexity. The treatment may involve combining restorative dentistry, endodontics and periodontics so that the teeth are retained in whole or in part. Such teeth can be useful as independent units of mastication or as abutments in simple fixed bridges. Continued periodontal breakdown may lead to total loss of tooth unless these defects can be repaired or eliminated and health of the tissues restored. Thus tooth resection procedures are used to preserve as much tooth structure as possible rather than sacrificing the whole tooth. ${ }^{1}$ The term tooth resection denotes the excision and removal of any segment of the tooth or a root with or without its accompanying crown portion. Various resection procedures described are: root amputation, hemisection, radisection and bisection. Root amputation refers to removal of one or more roots of multirooted tooth while other roots are retained. Hemisection denotes removal or separation of root with its accompanying crown portion of mandibular molars. Radisection is a newer terminology for removal of roots of maxillary molars. Bisection / bicuspidization is the separation of mesial and distal roots of mandibular molars along with its crown portion, where both segments are then retained individually. ${ }^{2}$

Weine $^{2}$ has listed the following indications for tooth resection

\section{Endodontic and Restorative Indications}

1) Prosthetic failure of abutments within a splint: If a single or multirooted tooth is periodontally involved within a fixed bridge,instead of removing the entire bridge, if the remaining abutment support is sufficient, the root of the involved tooth is extracted.27

2) Endodontic failure: Hemisection is useful in cases in which there is perforation through the floor of the pulp chamber, or pulp canal of one of the roots of an endodontically involved tooth which cannot be instrumented.

3) Vertical fracture of one root: The prognosis of vertical fracture is hopeless. If vertical fracture traverses one root while the other roots are unaffected, the offending root may be amputed.

4) Severe destructive process: This may occur as a result of furcation or sub. gingival caries, traumatic injury, and large root perforation during endodontic therapy.

Periodontal indications:

1) Severe vertical bone loss involving only one root of multi-rooted teeth.

2) Through and through furcation destruction.

3) Unfavourable proximity of roots of adjacent teeth, preventing adequate hygiene maintenance in proximal areas.

4) Severe root exposure due to dehiscence.

\section{Contraindications}

a) Strong adjacent teeth available for bridge abutments as alternatives to hemisection.

b) Inoperable canals in root to be retained. 


\section{International Journal of Science and Research (IJSR) \\ ISSN (Online): 2319-7064}

Index Copernicus Value (2013): 6.14 | Impact Factor (2014): 5.611

c) Root fusion-making separation impossible.

\section{Case Report}

A 35- years old male patient reported to the department of conservative and endodontics, Bhojia Dental College, Baddi, H.P., India, with the chief complaint of pain in lower right back teeth since past one week. Patient was relatively asymptomatic before then. He developed continuous and throbbing pain in this region, which got aggravated during mastication and sleep. Patient's medical history was not contributory.

On intra-oral examination, 46 was found to be grossly carious. On vertical percussion 46 was found to be sensitive. On probing, deep periodontal pocket was found in relation to 46. Vitality testing of 46 yielded no response. On radiographic examination (figure 1), grossly carious 46 was evident along with the furcation involvement and resorption of mesial root.

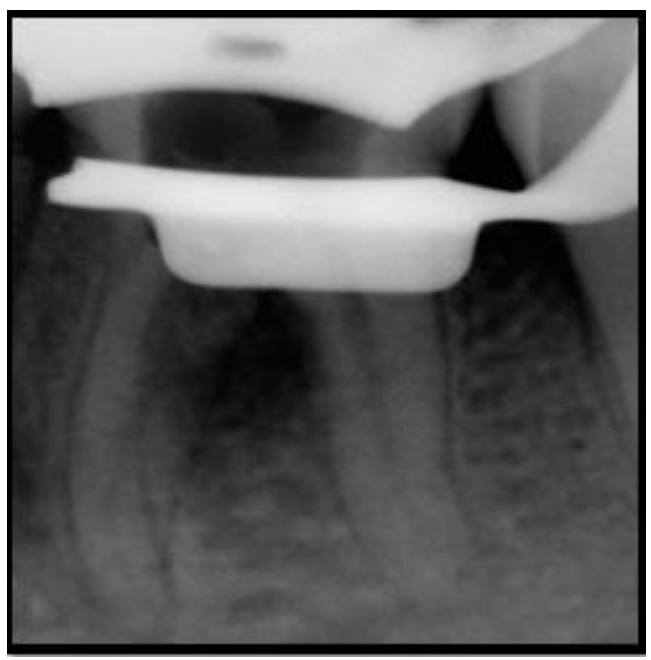

Figure 1

\section{Preoperative Radiograph}

In the view of above findings, it was decided to first carry out endodontic treatment of distal root of 46 followed by the hemisection of the mesial root of 46 while retaining- the distal root (as adequate bone support was present), followed by fabrication of crown and bridge over 45 and 47 using distal root of 46 as an abutment. After the proper obturation of distal root of 46, (Figure 2 ) hemisection was carried out in relation to mesial root of 46 , with the vertical cut method. A long shank, taper fissure carbide bur in high speed handpiece was placed along the buccal groove and a cut wasmade. The cut was channeled towards the center of the tooth and then directed towards the interproximal furcation opening of the mesial root. The bur was moved in the lingual and apical direction until the furcation area was reached. Once the bur had severed the floor of the pulp chamber, root was seperated from the remaining portion of the tooth (figure 3).A fine probe was passed through the cut to ensure separation. After seperation, the severed portion of the mesial root was removed with a extraction forceps (figure 4). The socket was irrigated with sterile saline to remove bony chips and debris.

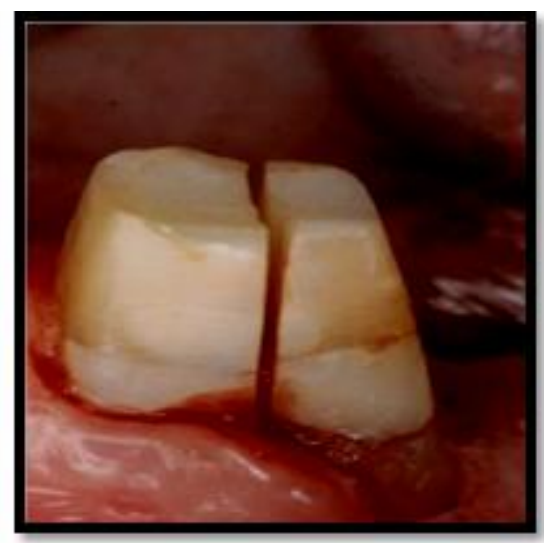

Figure 3: After Vertical Cut Made

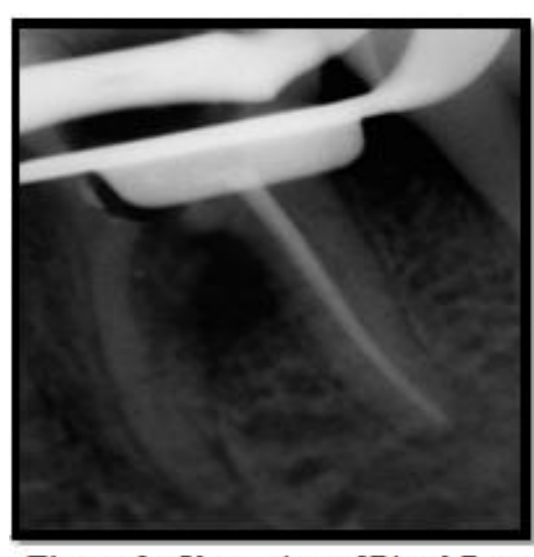

Figure 2: Obturation of Distal Root 


\section{International Journal of Science and Research (IJSR) \\ ISSN (Online): 2319-7064}

Index Copernicus Value (2013): 6.14 | Impact Factor (2014): 5.611

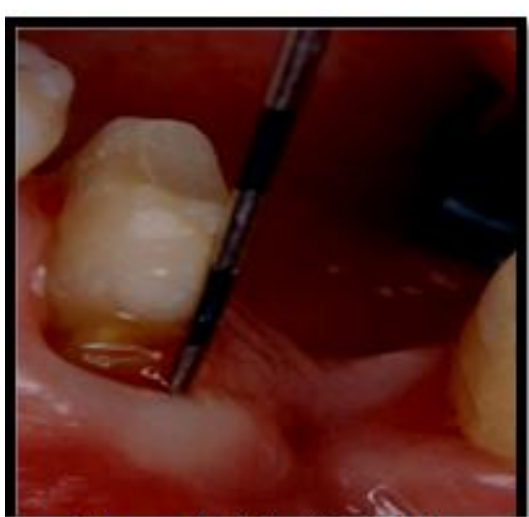

Figure 4: After Hemisection

The remaining portion of the distal tooth was trimmed to remove any ledges or sharp spicules, as these structures are potentially detrimental for periodontal maintenance. The occlusal table was minimized to redirect the forces. Bone graft material (perioglas, novabone products,usa) was placed.inside the socket of the mesial root. Silk sutures (trulon,sutures india pvt ltd) were placed to approximate the

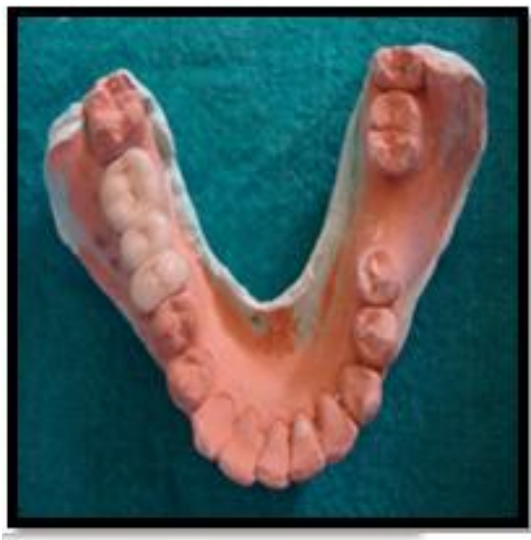

Figure 6: Fixed Prosthesis on Cast

\section{Discussion}

According to Buhler et al, Hemisection should be considered before every molar extraction, because this procedure can provide a good absolute biological cost savings with long term success. In addition, he reported that the failure rates of single tooth alloplastic (titanium) implants and hemisection are not substantially different. ${ }^{3}$ The decision of hemisectioning the tooth should be basis on the extent and pattern of bone loss, root trunk and root length, ability to eliminate the osseous defects and endodontic-restorative consideration. ${ }^{4}$ Consideration should also be given to morphology, clinical length and shape of the roots of a multirooted tooth. ${ }^{5}$ It is important to take into account the divergenc of the roots while making a case selection.

Affected teeth with roots spread apart facilitate the clinician's ability to carry out root resection. Teeth with closely approximated or fused roots are not good choices to receive hemisection therapy. ${ }^{5}$ Hemisection has been used to retain the teeth with furcation involvement. The tooth had to be endodontically treated before hemisection. In situations when resection periodontal therapy is decided, initiation of conventional endodontic treatment before therapy simplifies

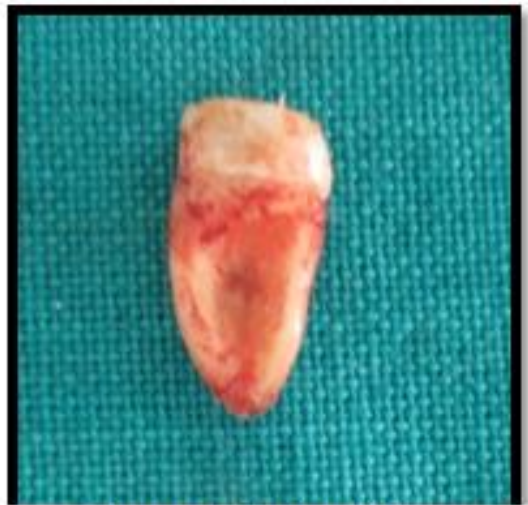

Figure 5: Sectioned Root

flap and periodontal pack (coe-pak, gc america inc.,usa) was applied. After 7 days, the sutures were removed. After healing of the tissues, fixed bridge involving retained distal half and Mandibular second premolar and second molar was given.(Figure 6,7)

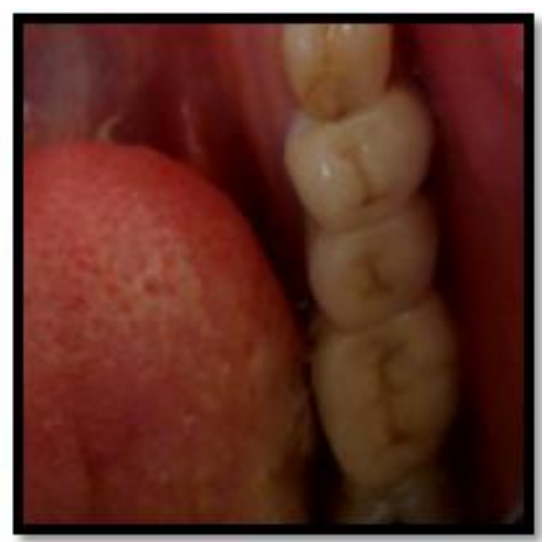

Figure 7: Cemented Fixed Prosthesis

the surgical procedure. This is because tooth preparation can invade the pulp chamber and jeopardize control of the coronal seal of the endodontic access opening complicating the completion of endodontic therapy. ${ }^{6}$

In the present case, because of excessive destruction of the mesial root due to the external root resorption and fair amount of the distal root remaining with adequate bone suppsort, hemisection carried out with the removal of the mesial root and crown. Remaining tooth structure was used as an abutment in crown and bridge after repositioning the occlusal contacts in favourable position.

Recently, Park et $a^{7}$ have suggested that hemisection of molars with questionable prognosis can maintain the teeth without detectable bone loss for a long-term period, provided that the patient has optimal oral hygiene. Saad et $a l^{8}$ have also concluded that hemisection of a mandibular molar may be a suitable treatment option when the decay is restricted to one root and the other root is healthy and remaining portion of tooth can very well act as an abutment. The prognosis for hemisection is the same as for routine endodontic procedures provided that case selection has been correct, the endodontics has been performed adequately, and the restoration is of an acceptable design relative to the 


\section{International Journal of Science and Research (IJSR) \\ ISSN (Online): 2319-7064}

Index Copernicus Value (2013): 6.14 | Impact Factor (2014): 5.611

occlusal and periodontal needs of the patient. The prognosis of root resected molars may not be as poor as previously believed. ${ }^{9}$ Multirooted ,periodontally involved molars can be maintainrd for long periods of time with hemisection. The large variation in success and failure reported by different authors is a reflection that roots resection and hemisection is a technique sensitive procedure. ${ }^{9}$ One must be careful throughout the processes of case selection and endodontic,periodontal,restorative and maintenance therapies. Critical analysis before reconstruction and regular revaluation during maintenance period are crucial. Langer et al reviewed records of 100 patients who had undergone root resection over a 10 years period. They reported a failure rate of $38 \%$ of which $15.8 \%$ occurred within the first 5 years after surgery. Most failures involved Mandibular teeth and occurred for reasons other than inflammatory periodontal disease. $^{10}$

Carnevale in his study on long term effects of root respective therapy suggested that it can be considered as an effective measure to resolve periodontal problems of furcation defects. ${ }^{11}$ The data indicate that recurrent periodontal disease is not a major cause of failure of these teeth. It was shown that such teeth can function successfully for long periods. Therefore early and correct diagnosis is imperative, as delay will result in rapid loss of supporting bone and eventually tooth loss.

\section{Conclusion}

Root amputation and hemisection should be considered as another weapon in the arsenal of the dental surgeon, determined to retain and not remove the natural teeth. With recent refinements in endodontics, periodontics and restorative dentistry, hemisection has received acceptance as a conservative and dependable dental treatment and teeth so treated have endured the demands of function. ${ }^{12}$ In conclusion, hemisection can be considred a suitable alternative to extraction and should be discussed with patients, during consideration of treatment options. The results of hemisection are predictable, and success rates are high if certain basic considerations are taken into account. ${ }^{5}$

\section{References}

[1] Basaraba N. Root Amputation and tooth hemisection.Dent Clin of N Amer 1969;13: 121

[2] Weine FS. Endodontic Therapy, 5th Edition.

[3] Buhler H. Survival Rates of hemisected Teeth : An Attempt to compare them with survival rates of alloplastic implants. Int J Periodontics Restorative Dent 1994;14(6).536-542.

[4] Newman,Takei, Fermin A Carranza. Furcation Involvement and Treatment; Willium F IN: Clinical Periodontology. X edition, WB Saunder's Co,200;996.

[5] Kurtzman GM, Silverstein LH, Shatz PC. Hemisection as an alternative treatment for vertically fractured Mandibular molars. Compend Contin Educ Dent.2006,27(2):126-29.

[6] 6 Farrar JN. Radical and Herioc treatment of alveolar abscess by amputation of roots of teeth. Dent Cosmos 1884,26:79-81
[7] Park JB. Hemisection of teeth with questionable prognosis. Report of a case with seven-year results; J Int Acad Periodontol. 2009; 11(3):214-9.

[8] Saad MN, Moreno J, Crawford C. Hemisection as an alternative treatment for decayed multirooted terminal abutment: a case report; J Can Dent Assoc. 2009 jun;75(5):387-90.

[9] Baston CH, Ammons WF.Persson R: Long term evaluation of root resected molars: A retrospective study: Int J Periodont Restor Dent 1996;16(3):207.

[10] Carnevale G, Difebo G, Toyelli MP: A retrospective analysis of the periodontal- Prosthetic treatment of molars with interradicular lesions; Int $\mathrm{J}$ Periodont Restor Dent 1991;11:188.

[11] Carnevale G, Pontoricro R, Di Febo G. Long Term Effect of root respective therapy in furcation involved molars- A 10 year longitudinal study. J Clin Periodontal 1998;25:209-14.

[12] Haueisen H, Heidemann D. Hemisection for treatment of an advanced endodontic-periodontal lesion: a case report. Int Endod J, 35, 557-572, 2002.

\section{List of Figures}

Figure 1 Preoperative Radiograph

Figure 2 Obturation of Distal Roo

Figure 3 After Vertical Cut Made

Figure 4 After Hemisection

Figure 5 Sectioned Root

Figure 6 Fixed Prosthesis on Cast

Figure 7 Cemented Fixed Prosthesis 